Research Paper

\title{
Healthcare-associated vancomycin resistant Enterococcus faecium infections in the Mansoura University Hospitals intensive care units, Egypt
}

\author{
Dalia Moemen, Doaa Tawfeek, Wafaa Badawy \\ Department of Medical Microbiology and Immunology, Faculty of Medicine, \\ Mansoura University, Mansoura, Egypt.
}

Submitted: May 12, 2014; Approved: December 31, 2014.

\begin{abstract}
Vancomycin resistant Enterococcus faecium (VREF) ia an emerging and challenging nosocomial pathogen. This study aimed to determine the prevalence, risk factors and clonal relationships between different VREF isolates in the intensive care units (ICUs) of the university hospitals in our geographic location. This prospective study was conducted from July, 2012 until September, 2013 on 781 patients who were admitted to the ICUs of the Mansoura University Hospitals (MUHs), and fulfilled the healthcare-associated infection (HAI) criteria. Susceptibility testing was determined using the disk diffusion method. The clonal relationships were evaluated with pulsed field gel electrophoresis (PFGE). Out of 52 E. faecium isolates, 12 (23.1\%) were vancomycin resistant. The significant risk factors for the VREF infections were: transfer to the ICU from a ward, renal failure, an extended ICU stay and use of third-generation cephalosporins, gentamicin, or ciprofloxacin. PFGE with the 12 isolates showed 9 different patterns; 3 belonged to the same pulsotype and another 2 carried a second pulsotypes. The similar pulsotypes isolates were isolated from ICUs of one hospital (EICUs); however, all of the isolates from the other ICUs had different patterns. Infection control policy, in conjunction with antibiotic stewardship, is important to combat VREF transmission in these high-risk patients.
\end{abstract}

Key words: Enterococcus faecium, ICU, vancomycin, risk factors, PFGE.

\section{Introduction}

Enterococci are opportunistic pathogens of the normal humans and animals intestinal microbiota. The most common Enterococcus species that is involved in nosocomial infections is Enterococcus faecium (E. faecium) (Top et al., 2008; Arias and Murray, 2012).

E. faecium has become one of the most important, emerging and challenging nosocomial pathogens (Arias and Murray, 2012). It is a difficult to treat this pathogen due to its intrinsic resistance to cephalosporins, aminoglycosides, clindamycin and trimethoprim-sulfamethoxazole (Leclercq et al., 2013). Moreover, it has the ability to easily acquire antibiotic resistance genes trough transfer of plasmids, chromosomal exchange or mutation (Jett et al., 1994).
In addition to this distinct resistance profile, genomic analyses have shown that hospital acquired E. faecium strains have a genetic repertoire that is distinct from that of community associated E. faecium strains that asymptomatically colonize the human gastrointestinal tract (van Schaik et al., 2010). This distinct genetic repertoire includes the enterococcal surface protein, Esp, which is a known virulence determinant (Heikens et al., 2007; 2011). Genomic islands that encode novel metabolic pathways (Heikens et al., 2008), and insertion sequence elements (Leavis et al., 2007). It is now considered that these determinants may be adaptive elements that have improved the relative fitness of $E$. faecium subpopulations in the hospital environment (Willems and van Schaik, 2009). Due to the resistance of multiple antibiotics, the treatment of choice in serious E. faecium infections is glycopeptides. However, prudent use of vancomycin is needed as it is associated with

Send correspondence to D. Moemen. Department of Medical Microbiology and Immunology, Faculty of Medicine, Mansoura University, Egypt. E-mail: dr_daliamoemen@yahoo.com. 
an increased risk for vancomycin resistant enterococci (VRE) colonization and infection (Tornieporth et al., 1996).

In the last decades, the number of VRE infections has increased (Grayson et al., 1991; Jones et al., 1995; Rice, 2001). The first VRE isolates were reported in the United Kingdom in the late 1980s (Uttley et al., 1988). In the United States, more than $80 \%$ of E. faecium isolates from hospitals are vancomycin resistant (Hidron et al., 2008). Vancomycin-resistant $E$. faecium (VREF) has been associated with outbreaks in hospitals worldwide (Arias and Murray, 2012). The VREF colonization and infection rates have risen steadily, with most cases being caused by the VanA and VanB genotypes, which are the most commonly encountered glycopeptide resistance forms (Coque et al., 1996; Rice, 2006; Deshpande et al., 2007).

The clinical impacts of VRE include the limited availability of drugs to treat VRE infections and the ability of VRE to transfer the genetic determinant for vancomycin resistance to other Gram-positive pathogens, such as Staphylococcus aureus (Schooneveld et al., 2008; Noble et al., 1992).

VREF is associated with hospital-acquired infections such as urinary tract infections, wounds, bacteremia, endocarditis and meningitis (Top et al., 2008; Arias and Murray, 2012). Several studies have demonstrated that VRE bacteraemia patients have a higher mortality rate than those infected with vancomycin-susceptible enterococci (Vergis et al., 2001; Edmond et al., 1996; Bhavnani et al., 2000).

Information regarding the VRE Infections prevalence in Egypt indicates that there is an increasing VRE infection rate. Ghonaim et al. (2009) reported that VRE isolates constituted $20.9 \%$ of hospital associated enterococcal infections at the Egyptian National Liver Institute.

To control the rapid spread of multidrug resistant organisms, it is necessary to understand the risk factors for acquiring them. Therefore, the aim of this study was to identify the VREF prevalence and possible risk factors in ICU patients who are at high risk of VREF infection. Additionally, we aimed to identify the clonal relationship between different isolates via pulsed-field gel electrophoresis (PFGE).

\section{Materials and Methods}

\section{Patients and methods}

\section{Study design}

This was a prospective study, which was conducted in nine ICUs in three different MUHs, that were situated within the same geographical region over a 15 months period from July, 2012 until September, 2013. The ICU bed numbers range from 4 to 27 , with a median of 10 . They ICUs were categorized as: A. emergency hospital ICUs (EICUs), B. specialized medical hospital ICUs (SMICUs) and C. children hospital ICUs, which included pediatric (PICU), neonatal ICU (NICU) and surgical ICU (SICU).

\section{Study population}

This study included 781 patients (who provided written consent) that were admitted to different MUHs ICUs and fulfilled the healthcare-associated infection (HAI) criteria. (Infections acquired $\geq 48$ hours after admission that were not present or incubating at the time of hospital admission). The infection types were documented with specific HAIs definitions, which were established by the Centers for Disease Control and Prevention (Horan et al., 2008).

\section{Data collection and definitions}

The following data were collected: demographic characteristics, ICU stay duration, transfer to the ICU from a ward, diagnosis at ICU admission, medical conditions that may alter the patient's immunity (comorbidity) (e.g. diabetes, malignancy, hepatic impairment, renal impairment and chronic lung diseases), invasive devices or procedures (e.g. surgical procedures, central venous catheter $[\mathrm{CVC}]$, peripheral venous catheter [PVC], mechanical ventilation, and total parenteral nutrition [TPN], drug therapy (e.g. antibiotics and their durations, prior antibiotic treatment, which was defined as any antibiotic treatment during the two weeks preceding ICU admission), immunosuppressive therapy (which included steroid therapy, cytotoxic chemotherapy or radiotherapy given within one month prior to ICU admission), and neutropenia (less than 500 neutrophils per $\mathrm{mm}^{3}$ )

A "case patient" was defined as patient who was infected with VREF and was attending a Mansoura University ICU during the study period. A "control patient" was defined as a Vancomycin susceptible Enterococcus faecium (VSEF) infected patient who was attending a Mansoura University ICU during the same period. The patients whose initial isolate was susceptible to vancomycin but who subsequently had VREF isolates that were recovered were included as case patients.

\section{Microbiologic studies}

The samples were processed in the Medical Diagnostics and Infection Control Unit (MDICU) laboratory using standard protocols. E. faecium laboratory identification was performed by Gram stain, colonial morphology on blood agar, growth and blackening of bile esculin agar, the absence of catalase production, resistance to $6.5 \%$ sodium chloride. The results were confirmed with the API 20 Strep (Biomerieux SA, Montalieu - Vercica and France) system. In vitro susceptibilities of the isolates to antimicrobials were determined with the disk diffusion method which was defined by the [CLSI] (Institute CaLS, 2011). The antibiotics that were tested included ampicillin $(10 \mu \mathrm{g})$, ciprofloxacin $(5 \mu \mathrm{g})$, high content gentamicin $(120 \mu \mathrm{g})$, erythromycin $(15 \mu \mathrm{g})$, tetracycline $(30 \mu \mathrm{g})$, vancomycin $(30 \mu \mathrm{g})$, 
teicoplanin $(30 \mu \mathrm{g})$, linezolid (30 $\mu \mathrm{g})$, and chloramphenicol $(30 \mu \mathrm{g})$. Glycopeptide antibiotics MICs for: vancomycin and teicoplanin against the E. faecium isolates were examined with the VITEK 2 system (bioMérieux).

PFGE

The clonal relationships between the vancomycinresistant strains were studied by evaluating the genomic DNA with PFGE (Antonishyn et al., 2000). DNA that was restricted with the SmaI enzyme was separated on an agarose gel using a CHEF DR III apparatus (Bio-Rad laboratories). The running conditions were $6 \mathrm{~V}$ per $\mathrm{cm}$, with pulses ranging from 2 to $15 \mathrm{~s}$ for $18 \mathrm{~h}$ at $14 \mu \mathrm{C}$. The DNA banding patterns were visualized under UV light after staining with ethidium bromide $(0.5 \mathrm{mg} / \mathrm{mL})$. The similarities between the isolates were determined by visual comparison of the isolate band patterns. The interpretation of the PFGE results was carried out by eye according to the criteria described by Tenover et al. (1995).

\section{Statistical analysis}

The SPSS software (SPSS Inc., Chicago, IL, USA) was used for data analyseis. Proportions were compared using the $\chi 2$ test and continuous variables were compared using Student's t or Mann-Whitney U tests. The results are presented as numbers (percentages) for frequency and as the mean \pm standard deviation for quantitative variables. Odds ratios (OR) $[95 \%$ confidence interval $(\mathrm{CI})]$ were calculated for all significant $(\mathrm{p}<0.05)$ qualitative variables in the univariate analyseis.

\section{Results}

\section{VREF Prevalence in the ICUs}

During the study period, a total of 975 specimens were collected from 782 patients, with clinically suspected HAIs in MUHs ICUs. Out of 52 E. faecium isolates detected, 12 were VREF, which constituted (12/52) $23.1 \%$ of the $E$. faecium isolates.

\section{VREF isolates}

Twelve VREF single-patient isolates (i.e., 1 isolate per patient) were obtained from the following sites: urine ( 7 isolates), blood ( 3 isolates) and wound ( 2 isolates). A total of 12 infections were documented in 12 patients, including urinary tract infection (7 patients), bacteremia instances (3 patients), and surgical site infection (2 patients). Of these 12 patients, 6 isolates were obtained from the emergency hospital ICUs, 4 were obtained from the ICUs of the specialized medical hospital and 2 were from PICUs.

\section{Antibiotic susceptibility testing of the VREF isolates}

Twelve E. faecium isolates were resistant to both glycopeptides antibiotics (vancomycin and teicoplanin) (with a vancomycin MIC $\geq 32 \mu \mathrm{g} / \mathrm{mL}$ ) (VanA phenotype).
All VREF isolates (100\%) were resistant to ampicillin, gentamicin, and ciprofloxacin. Most of the isolates (92\%) were resistant to erythromycin (only one isolate was sensitive), and 5 isolates were resistant to tetracycline ( $42 \%)$. All of the strains were sensitive to linezolid and chloramphenicol.

\section{The ICU-acquired VREF Risk factors}

Among the 52 E. faecium infected patients, 40 (76.9\%) had VSEF infections (control patients), and 12 (23.1\%) had VREF infections (case patients). The comparative demographic and clinical features for both the case and controls patients are listed in Tables 1 and 2. The risk factors that were significantly associated with the ICUacquired VREF infections were: transfer to the ICU from a ward $(p=0.04)$, renal failure $(p=0.01)$, and longer ICU stay duration $(p=0.018)$. Additionally, antibiotic use for a long period of time and use of third-generation cephalosporins, gentamicin, or ciprofloxacin were also associated with VREF infections.

\section{PFGE}

The restriction endonuclease patterns obtained with the PFGE following the SmaI treatment for the 12 VREF isolates are presented in Figure 1. In general, the 12 isolates showed 9 different patterns. Three isolates belonged to the same pulsotype and another 2 carried similar pulsotypes. Of interest, the isolates with similar pulsotypes were isolated from the ICUs of one hospital (EICUs); however, all of the isolates from the other ICUs had different patterns.

\section{Discussion}

E. faecium is a highly resistant nosocomial pathogen and has recently emerged as an important threat in hospitals worldwide (Arias and Murray, 2012). In this study, VREF constituted $23.1 \%$ of the observed E. faecium isolates, a result that was close to the Ghonaim et al. study observations, in which the VRE isolates constituted $20.9 \%$ of the hospital-associated enterococcal infections at the National Liver Institute (Ghonaim et al., 2009), which indicated a big problem in Egypt. Similar studies from the United States reported vancomycin resistance in up to $28 \%$ of all nosocomial Enterococcus species (NNIS, 2004). However studies from India and South America showed much lower of VRE infection prevalence ranging from 1- 8.7\% (Mathur et al., 2003; Taneja et al., 2004; Kapoor et al., 2005; Kaur et al., 2009; Praharaj et al., 2003; Panesso et al., 2013). E. faecium antibiotics sensitivity pattern in the present study is consistent with the concept that vancomycin resistance is usually accompanied by resistance to other antimicrobial agents, such as penicillin, ampicillin, erythromycin, ciprofloxacin and gentamicin. The objective of this study was to determine the risk factors for HAI that were associated with VREF among the MUH ICU inpatients. We found that transfers to the ICU from a ward $(\mathrm{p}=0.04)$ and renal fail- 
Table 1 - ICU-acquired VREF infection risk factors.

\begin{tabular}{|c|c|c|c|c|c|c|}
\hline \multirow[t]{3}{*}{ Factor } & \multicolumn{4}{|c|}{ Patients with $E$. faecium infections } & \multirow[t]{3}{*}{$\mathrm{p}$ - value } & \multirow[t]{3}{*}{ Odds ratio } \\
\hline & \multicolumn{2}{|c|}{ VREF $\mathrm{n}=12$} & \multicolumn{2}{|c|}{ VSEF $\mathrm{n}=40$} & & \\
\hline & No. & $(\%)$ & No. & $(\%)$ & & \\
\hline Sex (male): & 7 & 58.3 & 25 & 62.5 & 0.8 & 0.84 \\
\hline Diabetes mellitus & 3 & 25.0 & 7 & 17.5 & 0.7 & 1.6 \\
\hline Neutropenia & 4 & 33.3 & 10 & 25.0 & 0.7 & 1.5 \\
\hline Neoplastic disease & 5 & 41.7 & 16 & 40.0 & 0.9 & 1.1 \\
\hline Transfer to the ICU from a ward & 8 & 66.6 & 12 & 30.0 & $0.04 *$ & 4.7 \\
\hline Surgery & 4 & 33.3 & 14 & 35.0 & 1 & 0.9 \\
\hline Immunosuppressive drugs & 5 & 41.7 & 11 & 27.5 & 0.4 & 1.9 \\
\hline Prior antibiotic treatment & 10 & 83.3 & 28 & 70.0 & 0.5 & 2.1 \\
\hline \multicolumn{7}{|l|}{ Organ failure: } \\
\hline Respiratory & 3 & 25 & 6 & 15 & 0.4 & 1.9 \\
\hline Cardiac & 2 & 16.7 & 7 & 17.5 & 1 & 0.9 \\
\hline Renal & 5 & 41.7 & 3 & 7.5 & $0.01 *$ & 8.8 \\
\hline Neurologic & 1 & 8.3 & 6 & 15.0 & 1 & 0.5 \\
\hline Liver & 3 & 25 & 5 & 12.5 & 0.4 & 2.3 \\
\hline \multicolumn{7}{|l|}{ During ICU hospitalization: } \\
\hline Intravascular catheter & 12 & 100 & 36 & 90 & 0.6 & - \\
\hline Urinary catheter & 12 & 100 & 37 & 92.5 & 1 & - \\
\hline Mechanical ventilation & 12 & 100 & 37 & 92.5 & 1 & - \\
\hline ICU stay duration (days) & \multicolumn{2}{|c|}{$12 \pm 5.51$} & \multicolumn{2}{|c|}{$9.23 \pm 4.86$} & $0.018 *$ & $0.47-5.06$ \\
\hline
\end{tabular}

* Significant, p-value $\leq 0.05$; ICU, intensive care unit.

Table 2 - Antibiotic use amongst the study patients during their ICU stays.

\begin{tabular}{|c|c|c|c|c|c|c|}
\hline \multirow[t]{3}{*}{ Factor } & \multicolumn{4}{|c|}{ Patients with $E$. faecium infections } & \multirow[t]{3}{*}{$\mathrm{p}$-value } & \multirow[t]{3}{*}{ Odds ratio } \\
\hline & \multicolumn{2}{|c|}{ VREF $\mathrm{n}=12$} & \multicolumn{2}{|c|}{ VSEF $\mathrm{n}=40$} & & \\
\hline & No. & $(\%)$ & No. & $(\%)$ & & \\
\hline Antibiotic treatment duration, days & $16 \pm 11$ & & $5 \pm 6$ & & $<0.001 * *$ & 8.09-13.91 \\
\hline Vancomycin use & 5 & 41.7 & 12 & 30.0 & 0.45 & 1.66 \\
\hline Extended-spectrum penicillin use & 7 & 58.3 & 19 & 47.5 & 0.5 & 1.6 \\
\hline Fluoroquinolone use & 9 & 75 & 16 & 40.0 & 1 & 0.8 \\
\hline Carbapenem use & 3 & 25.0 & 12 & 30.0 & 1 & 0.8 \\
\hline Aminoglycoside use & 6 & 50.0 & 8 & 20.0 & $0.04 *$ & 4 \\
\hline First generation cephalosporin use & 2 & 16.7 & 18 & 45.0 & 0.09 & 0.2 \\
\hline Second generation cephalosporin use & 2 & 16.7 & 15 & 37.5 & 0.3 & 0.3 \\
\hline Third generation cephalosporin use & 6 & 50.0 & 6 & 15.0 & $0.01 *$ & 5.7 \\
\hline
\end{tabular}

*Significant, p-value $\leq 0.05 ; *$ highly significant, $\mathrm{p}$-value $\leq 0.001$.

ures $(\mathrm{P}=0.01)$ were associated with VREF infections. Intra hospital transfer was associated with VREF colonization or infection in other studies (Tornieporth et al., 1996; Webb et al., 2001). The patients may have acquired E. facium during their ward stay, which later caused infections during their ICU stays. However, our study showed that renal failure was a risk factor for VREF infection acquisition. This is dif- ferent from other studies in which dialysis was not associated with the VREF infection incidences (Webb et al., 2001; Handwerger et al., 1993; Descheemaeker et al., 2000). Patients who require hemodialysis often have complicated illnesses and may receive multiple antibiotic courses, including vancomycin, which places them at greater risk for VREF infection or colonization. Frequent hospital- 


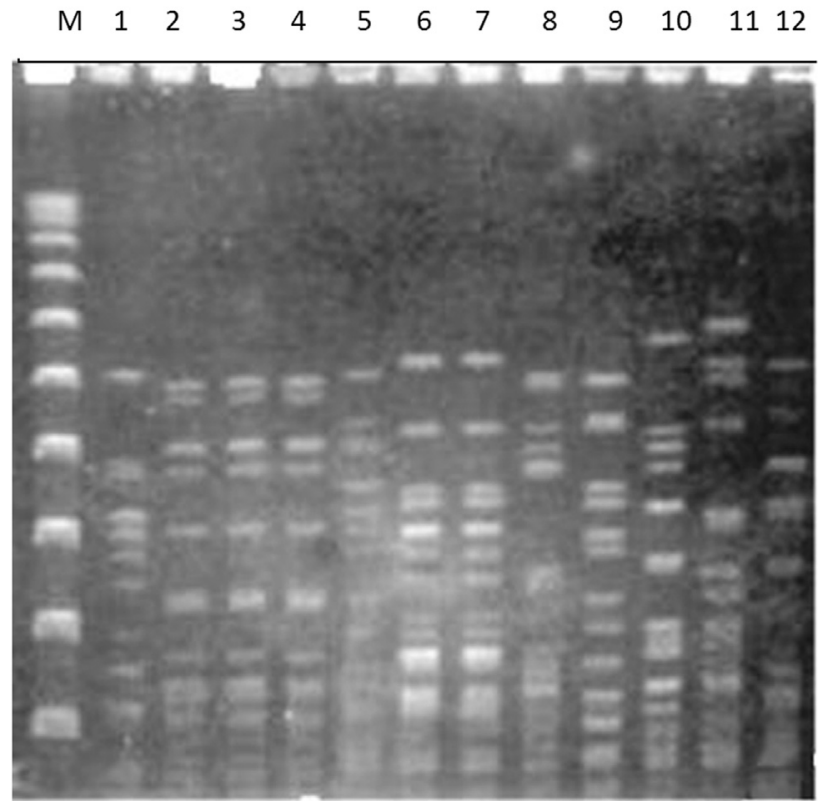

Figure 1 - SmaI restriction endonuclease patterns obtained by PFGE: Lane M marker; lane 1, patient 1 (SMICU); lanes 2, 3and 4 (EICUs); lane 5, (PICU); lanes 6 and 7, (EICUs); lane 8, (PICU); lane 9, (SMICU); lane 10, (SMICU); lane 11, (SMICU); lane 12, (EICU).

izations and cross-transmission can also contribute to VREF infection in patients undergoing hemodialysis (Chow et al., 1993; Roghmann et al., 1998). The other risk factor for VREF infection in the ICU was longer ICU stay duration ( $\mathrm{p}=0.018)$. This result consistant with most previous studies (Ostrowsky et al., 1999; Warren et al., 2003; Se et al., 2009; Pan et al., 2012; Fridkin et al., 2001). Longer ICU stays can indicate a greater chance of receiving antibiotics and also a longer exposure time to possible pathogen transmission. Our study showed that patients who had a prior exposure to broad-spectrum antibiotics and for a prolonged duration were at a higher risk of VREF infections due to the selective pressure from prior antibiotics. Interestingly, in this study, vancomycin use was not found to be a risk factor for VREF infection, which is different from other studies (Tornieporth et al., 1996; Fridkin et al., 2001). This can be explained by the theory that in medical settings in which VREF infections are endemic, the patients with VREF colonization may serve as a source for already antibiotic resistant $E$. faecium strains in patients who have not necessarily received glycopeptide antibiotics (Murray, 2000; Hayden, 2000). According to the PFGE analysis, there were nine pulsotypes that were noted during the study period. The PFGE analysis showed different patterns in the different ICUs of the three hospitals, except for the EICUs in the emergency hospital, in which the 6 VREF isolates showed 3 different patterns. Specifically, 3 isolates belonged to the same pulsotype and another 2 carried similar pulsotypes, indicating that there was a spread within the ICUs of one hospital (the emergency hospital). Thus, our data show that all of the strains were not from the same clone, indicating multiple acquisitions of resistant isolates. However, the VREF infections within the ICUs of one hospital (the EICUs) may result from cross transmission of prevalent isolates. This shows that spread occurred within the ICUs of an individual hospital but not in the other hospitals. This might be because the EICUs have more critical care cases compared with the other ICUs (e.g., care for patients with acute, life-threatening illnesses or injuries), in which VRE infection prevention measures are more important than in the other ICU type's. Similarly other studies have documented the spread of VREF clones among hospitals (Sader et al., 1994; Fridkin et al., 1998; Nourse et al., 2000; Corso et al., 2007). The microorganism can be transmitted by health care workers in particular via their hands which are most likely the most common mode of nosocomial transmission (Boyce et al., 1994). VREF transmission by way of contaminated medical equipment and health care carriers has been investigated and shown to occur in different hospitals sections especially in the dialysis ward (Kalocheretis et al., 2004). Adherence to infection control precautions by ICU staff members may also affect possible VREF transmission. Other distinct strains may come from intrinsic E. facium, which is selected for under selective pressure. Thus, infection control policy, in conjunction with practices that control antimicrobial use, is important to combat VREF infection transmission in these high-risk patients. Moreover, our data ensure that standard efforts to reduce cross-transmission might be needed hospital-wide because the VREF rates outside of the ICUs greatly affect the ICU- specific VREF rates.

\section{References}

Antonishyn NA, McDonald RR, Chan E et al. (2000) Evaluation of fluorescence-based amplified fragment length polymorphism analysis for molecular typing in hospital epidemiology: comparison with pulsed-field gel electrophoresis for typing strains of vancomycin resistant Enterococcus faecium. J Clin Microbiol 38:4058-4065.

Arias CA, Murray BE (2012) The rise of the Enterococcus: beyond vancomycin resistance. Nat Rev Microbiol 10:266278.

Bhavnani SM, Drake JA, Forrest A et al. (2000) A nationwide, multicenter, case-control study comparing risk factors, treatment, and outcome for vancomycin-resistant and -susceptible enterococcal bacteremia. Diagn Microbiol Infect Dis 36:145-158.

Boyce JM, Opal SM, Chow JW et al. (1994) Outbreak of multidrug-resistant Enterococcus faecium with transferable vanB class vancomycin resistance. J Clin Microbiol 32:11481153.

Chow JW, Kuritza A, Shlaes DM et al. (1993) Clonal spread of vancomycin- resistant Enterococcus faecium between patients in three hospitals in two states. J Clin Microbiol 31:1609-1611.

Coque TM, Tomayko JF, Ricke SC et al. (1996) Vancomycinresistant enterococci from nosocomial, community, and ani- 
mal sources in the United States. Antimicrob Agents Chemother 40:2605-2609.

Corso AC, Gagetti PS, Rodríguez MM et al. (2007) Molecular epidemiology of vancomycin-resistant Enterococcus faecium in Argentina. Int J Infect Dis 11:69-75.

Descheemaeker P, Ieven M, Chapelle S et al. (2000) Prevalence and molecular epidemiology of glycopeptide-resistant enterococci in Belgian renal dialysis units. J Infect Dis 181:235-241.

Deshpande LM, Fritsche TR, Moet GJ et al. (2007) Antimicrobial resistance and molecular epidemiology of vancomycinresistant enterococci from North America and Europe: a report from the SENTRY antimicrobial surveillance program. Diagn Microbiol Infect Dis 58:163-170.

Edmond MB, Ober JF, Dawson JD et al. (1996) Vancomycin-resistant enterococcal bacteremia: Natural history and attributable mortality. Clin Infect Dis 23:1234-1239.

Fridkin SK, Edwards JR, Courval JM et al. (2001) Intensive Care Antimicrobial Resistance Epidemiology (ICARE) Project and the National Nosocomial Infections Surveillance (NNIS) System Hospitals. The effect of vancomycin and third-generation cephalosporins on prevalence of vancomycin-resistant enterococci in 126 U.S. adult intensive care units. Ann Intern Med 135:175-183.

Fridkin SK, Yokoe DS, Whitney CG et al. (1998) Epidemiology of a dominant clonal strain of vancomycin-resistant Enterococcus faecium at separate hospitals in Boston, Massachusetts. J Clin Microbiol 36:965-970.

Ghonaim M, Ghoniem E, Abdulaziz A et al. (2009) Enterococci in Hospital Associated Infection in the National Liver Institute, Egypt. Egy J Med Microbiol 18:69-79.

Grayson ML, Eliopoulos GM, Wennersten CB et al. (1991) Increasing resistance to beta-lactam antibiotics among clinical isolates of Enterococcus faecium: a 22-year review at one institution. Antimicrob Agents Chemother 35:2180-2184.

Handwerger S, Raucher B, Altarac D et al. (1993) Nosocomial outbreak due to Enterococcus faecium highly resistant to vancomycin, penicillin, and gentamicin. Clin Infect Dis 16:750-755.

Hayden MK (2000) Insights into the epidemiology and control of infection with vancomycin-resistant enterococci. Clin Infect Dis 31:1058-1065.

Heikens E, Bonten MJ, Willems RJ (2007) Enterococcal surface protein Esp is important for biofilm formation of Enterococcus faecium E1162. J Bacteriol 189:8233-8240.

Heikens E, Singh KV, Jacques-Palaz KD et al. (2011) Contribution of the enterococcal surface protein Esp to pathogenesis of Enterococcus faecium endocarditis. Microbes Infect 13:1185-1190.

Heikens E, van Schaik W, Leavis HL et al. (2008) Identification of a novel genomic island specific to hospital-acquired clonal complex 17 Enterococcus faecium isolates. Appl Environ Microbiol 74:7094-7097.

Hidron AI, Edwards JR, Patel J et al. (2008) NHSN annual update: antimicrobial-resistant pathogens associated with healthcare-associated infections: annual summary of data reported to the national healthcare safety network at the centers for disease control and prevention, 2006-2007. Infect Control Hosp Epidemiol 29:996-1011.

Horan TC, Andrus M, Dudeck MA (2008) CDC/NHSN surveillance definition of health care-associated infection and crite- ria for specific types of infections in the acute care setting. Am J Infect Control 36:309-332.

Institute CaLS (2011) Performance standards for antimicrobial susceptibility testing: 17th informational supplement, M100-S21. Wayne, PA.:Clinical and Laboratory Standards Institute.

Jett BD, Huycke MM, Gilmore MS (1994) Virulence of enterococci. Clin Microbiol Rev 7:462-478.

Johnson AP, Uttley AH, Woodford N et al. (1990) Resistance to vancomycin and teicoplanin: an emerging clinical problem. Clin Microbiol Rev 3:280-291.

Jones RN, Sader HS, Erwin ME et al. (1995) Emerging multiply resistant enterococci among clinical isolates. I. Prevalence data from 97 medical center surveillance study in the United States. Enterococcus Study Group. Diagn Microbiol Infect Dis 21:85-93.

Kalocheretis P, Baimakou E, Zerbala S et al. (2004) Dissemination of vancomycin-resistant enterococci among haemodialysis patients in Athens, Greece. J Antimicrob Chemother 54:1031-1034.

Kapoor L, Randhawa VS, Deb M (2005) Antimicrobial resistance of enterococcal blood isolates at a pediatric care hospital in India. Jpn J Infect Dis 58:101-103.

Kaur N, Chaudhary U, Aggarwal R et al. (2009) Emergence of VRE and their antimicrobial sensitivity pattern in a tertiary care teaching hospital. J Med Biol Sci 8:26-32.

Leavis HL, Willems RJ, van Wamel WJ et al. (2007) Insertion sequence-driven diversification creates a globally dispersed emerging multiresistant subspecies of E. faecium. PLoS Pathog 3:75-96.

Leclercq R, Canton R, Brown DF et al. (2013) EUCAST expert rules in antimicrobial susceptibility testing. Clin Microbiol Infect 19:141-160.

Mathur P, Kapil A, Chandra R et al. (2003) Antimicrobial resistance in Enterococcus faecalis at a tertiary care centre of northern India. Indian J Med Res 118:25-28.

Murray BE (2000) Vancomycin-resistant enterococcal infections. N Engl J Med 342:710-721.

NNIS (2004) National Nosocomial Infections Surveillance (NNIS) System Report, data summary from January 1992 through June 2004, issued October 2004. Am J Infect Control 32:470-485.

Noble WC, Virani Z, Cree RG (1992) Co-transfer of vancomycin and other resistance genes from Enterococcus faecalis NCTC 12201 to Staphylococcus aureus. FEMS Microbiol Lett 72:195-198.

Nourse C, Byrne C, Kaufmann M et al. (2000) VRE in the Republic of Ireland: clinical significance, characteristics and molecular similarity of isolates. J Hosp Inf 44:288-293.

Ostrowsky BE, Venkataraman L, D’Agata EMC et al. (1999) Vancomycin-Resistant Enterococci in Intensive Care Units: High Frequency of Stool Carriage During a Non-Outbreak Period. Arch Intern Med 159:1467-1472.

Pan SC, Wang JT, Chen YC et al. (2012) Incidence of and risk factors for infection or colonization of vancomycin-resistant enterococci in patients in the intensive care unit. PLoS ONE 7:e47297.

Panesso D1, Reyes J, Rincón S et al. (2010) Molecular epidemiology of vancomycin-resistant Enterococcus faecium: a prospective, multicenter study inSouth American hospitals. J Clin Microbiol 48:1562-1569. 
Praharaj I, Sujatha S, Parija SC (2013) Phenotypic \& genotypic characterization of vancomycin resistant Enterococcus isolates from clinical specimens. Indian J Med Res 138:549556.

Rice LB (2001) Emergence of vancomycin-resistant enterococci. Emerg Infect Dis 7:183-187.

Rice LB (2006) Antimicrobial resistance in gram-positive bacteria. Am J Med 119:11-19. Roghmann MC, Fink JC, Polish L et al. (1998) Colonization with vancomycin-resistant enterococci in chronic hemodialysis patients. Am J Kidney Dis 32:254-257.

Sader HS, Pfaller MA, Tenover FC et al. (1994) Evaluation and characterization of multiresistant Enterococcus faecium from 12 U.S. medical centers. J Clin Microbiol 32:28402842 .

Schooneveld TV, Rupp ME (2010) Control of Gram-positive multidrug-resistant pathogens. In: Lautenbach E, Woeltje KF, Malani PN, editors. Practical healthcare epidemiology. 3rd ed. Chicago, United States: The University of Chicago Press p. 197-208.

Se YB, Chun HJ, Yi HJ et al. (2009) Incidence and risk factors of infection caused by vancomycin-resistant enterococcus colonization in neuro- surgical intensive care unit patients. $\mathrm{J}$ Korean Neurosurg Soc 46:123-129.

Taneja N, Rani P, Emmanuel R et al. (2004) Significance of vancomycin resistant enterococci from urinary specimens at a tertiary care centre in northern India. Indian J Med Res 119:72-74.

Tenover FC, Arbeit RD, Goering RV et al. (1995) Interpreting chromosomal DNA restriction patterns produced by pulsed-field gel electrophoresis: criteria for bacterial strain typing. J Clin Microbiol 33:2233-2239.
Top J, Willems R, Bonten M (2008) Emergence of CC17 Enterococcus faecium: from commensal to hospital-adapted pathogen. FEMS Immunol Med Microbiol 52:297-308.

Tornieporth NG, Roberts RB, John J et al. (1996) Risk factors associated with vancomycin-resistant Enterococcus faecium infection or colonization in 145 matched case patients and control patients. Clin Infect Dis 23:767-772.

Tornieporth NG, Roberts RB, John J et al. (1996) Risk factors associated with vancomycin-resistant Enterococcus faecium infection or colonization in 145 matched case patients and control patients. Clin Infect Dis 23:767-772.

Uttley AH, Collins CH, Naidoo J et al. (1988) Vancomycinresistant enterococci. Lancet 1:57-58.

van Schaik W, Top J, Riley DR et al. (2010) Pyrosequencingbased comparative genome analysis of the nosocomial pathogen Enterococcus faecium and identification of a large transferable pathogenicity island. BMC Genomics 11:239.

Vergis EN, Hayden MK, Chow JW et al. (2001) Determinants of vancomycin resistance and mortality rates in enterococcal bacteremia. A prospective multicenter study. Ann Intern Med 135:484-492.

Warren DK, Kollef MH, Seiler S et al. (2003) The Epidemiology of Vancomycin-Resistant Enterococcus Colonization in a Medical Intensive Care Unit Infect Control Hosp Epidemiol 24:257-263.

Webb M, Riley LW, Roberts RB (2001) Cost of hospitalization for and risk factors associated with vancomycin-resistant Enterococcus faecium infection and colonization. Clin Infect Dis 33:445-452.

Willems RJ, van Schaik W (2009) Transition of Enterococcus faecium from commensal organism to nosocomial pathogen. Future Microbiol 4:1125-1135.

\section{Associate Editor: Roxane Maria Fontes Piazza}

All the content of the journal, except where otherwise noted, is licensed under a Creative Commons License CC BY-NC. 